\title{
Modelling and Numerical Simulation of the Dynamics of Glaciers Including Local Damage Effects
}

\author{
G. Jouvet ${ }^{1}{ }^{*}$, M. Picasso ${ }^{1}$, J. Rappaz ${ }^{1}$, M. Huss ${ }^{2}$ and M. Funk ${ }^{3}$ \\ ${ }^{1}$ Mathematics Institute of Computational Science and Engineering, EPFL \\ 1015 Lausanne, Switzerland \\ ${ }^{2}$ Department of Geosciences, University of Fribourg, 1700 Fribourg, Switzerland \\ ${ }^{3}$ Laboratory of Hydraulics, Hydrology and Glaciology (VAW), ETHZ, 8092 Zurich, Switzerland
}

\begin{abstract}
A numerical model to compute the dynamics of glaciers is presented. Ice damage due to cracks or crevasses can be taken into account whenever needed. This model allows simulations of the past and future retreat of glaciers, the calving process or the break-off of hanging glaciers. All these phenomena are strongly affected by climate change.

Ice is assumed to behave as an incompressible fluid with nonlinear viscosity, so that the velocity and pressure in the ice domain satisfy a nonlinear Stokes problem. The shape of the ice domain is defined using the volume fraction of ice, that is one in the ice region and zero elsewhere. The volume fraction of ice satisfies a transport equation with a source term on the upper ice-air free surface accounting for ice accumulation or melting. If local effects due to ice damage must be taken into account, the damage function $D$ is introduced, ranging between zero if no damage occurs and one. Then, the ice viscosity $\mu$ in the momentum equation must be replaced by $(1-D) \mu$. The damage function $D$ satisfies a transport equation with nonlinear source terms to model cracks formation or healing.

A splitting scheme allows transport and diffusion phenomena to be decoupled. Two fixed grids are used. The transport equations are solved on an unstructured grid of small cubic cells, thus allowing numerical diffusion of the volume fraction of ice to be reduced as much as possible. The nonlinear Stokes problem is solved on an unstructured mesh of tetrahedrons, larger than the cells, using stabilized finite elements.

Two computations are presented at different time scales. First, the dynamics of Rhonegletscher, Swiss Alps, are investigated in 3D from 2007 to 2100 using several climatic scenarios and without considering ice damage. Second, ice damage is taken into account in order to reproduce the calving
\end{abstract}

\footnotetext{
${ }^{*}$ Corresponding author. E-mail: guillaume.jouvet@gmail.com
} 
process of a $2 \mathrm{D}$ glacier tongue submerged by water.

Key words: free surface flows, finite elements, volume of fluid, damage mechanics, glaciers, calving

AMS subject classification: 76A05, 76D27

\section{Introduction}

During the last century, a dramatic retreat of Alpine glaciers has been observed [8, 24]. This phenomena is expected to last throughout the next century and will have severe impacts on the landscape and tourism in the Alps, water supply in summer and hydropower production, but also on risk management of natural hazards (e.g. ice avalanches or glacier floods). Numerical simulation is a valuable tool to understand how climate change affects alpine glaciers and can provide answers to practical questions, such as the speed of glacier retreat over the next century, or the timing of the break-off of hanging glaciers and glacier tongues terminating in water.

In this paper, the free boundary problem that consists in finding the shape of a glacier over a given period and under given climatic conditions is addressed. When considering the dynamics of glaciers during years or decades, ice can be considered as an incompressible Non Newtonian fluid, flowing along the bedrock since subject to gravity forces [10]. Ice accumulates at the top of the glacier due to snowfall exceeding melt whereas melt dominates in the lower parts of the glacier.

When the goal is to reproduce the dynamics of a complete glacier as in Fig. 1a the formation of ice cracks and crevasses does not need to be modelled. Indeed, for instance in $[14,15,16]$, results in good agreement with observations have been obtained for Alpine glaciers during centuries, without modelling ice damage. However, when local effects have to be considered, such as the collapse of a hanging glacier or the calving process [2] (see Fig. 1b), then the modelling of ice damage cannot be avoided. In [19, 20, 21, 22] a model to account for the formation of ice cracks has been proposed and solved numerically using the level set method. The goal of this study is to incorporate this model into the numerical model presented in $[14,15,16]$.

The model for undamaged glaciers has already been presented in $[15,16]$ and is based on the following procedure: Given the shape of the glacier, the velocity and pressure in the ice region satisfy a nonlinear Stokes problem. An Eulerian framework is considered and the ice region is defined using the volume fraction of ice, one in the ice region, zero elsewhere, as for the Volume of Fluid (VOF) method [23]. The volume fraction of ice satisfies a transport equation with a source term on the ice-air free surface to account for snow accumulation or melting.

In order to model ice cracks, a damage field $D$, accounting for the progressive deterioration of ice was introduced in $[19,20,21,22]$. The damage ranges between zero (no damage) and one (full damage). Given the velocity and pressure fields, $D$ satisfies a transport equation with source terms to account for the formation of cracks. Then, the viscosity $\mu$ in the momentum equation must be replaced by $(1-D) \mu$ when damage is considered. 
Two applications demonstrate the potential of the numerical model presented in this paper. In a first study case, the dynamics of Rhonegletscher, a valley glacier in the Swiss Alps, is simulated until 2100. using simple assumptions on future climate. Unlike [15], we consider new scenarios that are independent of predictions based on climate model results. For this experiment damage is not taken into account. The second application consists in simulating the calving process in a simplified $2 \mathrm{D}$ ice slab.

This paper is organized as follows: the model corresponding to a damaged and undamaged ice flow is presented in the next Section. Section 3 is devoted to the numerical method. Section 4 describes the simulation of Rhonegletscher without damage. Finally, a simulation of the calving process is presented in Section 5.

\section{Modelling}

Let $\Lambda$ be a cavity of $\mathbb{R}^{d}, d=2,3$, containing the ice domain $\Omega(t)$ at time $t, 0 \leq t \leq T$. When $d=2, \Lambda=[0, \bar{X}] \times[0, \bar{Z}]$, when $d=3, \Lambda=[0, \bar{X}] \times[0, \bar{Y}] \times[0, \bar{Z}]$, see Fig. 1d and 1c, respectively.

\subsection{Undamaged glacier velocity}

When considering the motion of a glacier during years, ice can be considered as an extremely viscous and incompressible non-Newtonian fluid. The mass and momentum conservation equations reduce at time $t$ to a stationary nonlinear Stokes problem in the ice domain:

$$
\begin{aligned}
-2 \operatorname{div}(\mu \varepsilon(\mathbf{u}))+\nabla p & =\rho \mathbf{g} & & \text { in } \Omega(t), \\
\operatorname{div} \mathbf{u} & =0 & & \text { in } \Omega(t),
\end{aligned}
$$

where $\mathbf{u}$ is the ice velocity, $p$ the ice pressure, $\mu$ the ice viscosity, $\varepsilon(\mathbf{u})=\frac{1}{2}\left(\nabla \mathbf{u}+\nabla \mathbf{u}^{T}\right)$ the rate of strain tensor, $\rho$ the ice density and $\mathrm{g}$ the gravity. Here above, the viscosity $\mu$ is a function of $\varepsilon(\mathbf{u})$ and satisfies an implicit relation, the so-called Glen's law $[9,10,16]$ :

$$
\frac{1}{2 \mu}=A\left(\tau_{0}^{m-1}+(2 \mu|\varepsilon(\mathbf{u})|)^{m-1}\right)
$$

where $A$ is a positive number known as the rate factor, $m \geq 1$ is Glen's exponent, $\tau_{0}>0$ is a small regularization parameter and $|\varepsilon(\mathbf{u})|:=\sqrt{\frac{1}{2} \varepsilon(\mathbf{u}): \varepsilon(\mathbf{u})}$. When $m>1$, it is shown in [14] that $\mu$ is positive and strictly decreasing with respect to $|\varepsilon(\mathbf{u})|$. Note that $\tau_{0}=0$ in the original Glen's law yields to an infinite viscosity at 0 . When $\tau_{0}>0$, the viscosity is finite at 0 and has the following asymptotic behaviour when $|\varepsilon(\mathbf{u})|$ goes to the infinity:

$$
\mu(|\varepsilon(\mathbf{u})|)=\mathcal{O}\left(|\varepsilon(\mathbf{u})|^{\frac{1}{m}-1}\right) .
$$

When $m=1$, then $\mu$ is constant and the problem (2.1) (2.2) corresponds to a Newtonian fluid. In the framework of glaciology, $m$ is often taken equal to 3, see [11], then equation (2.3) can 


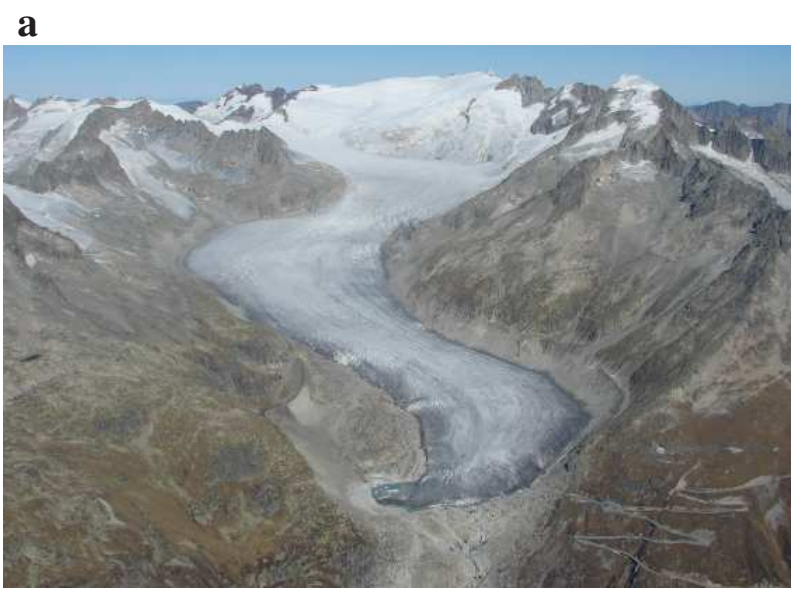

b
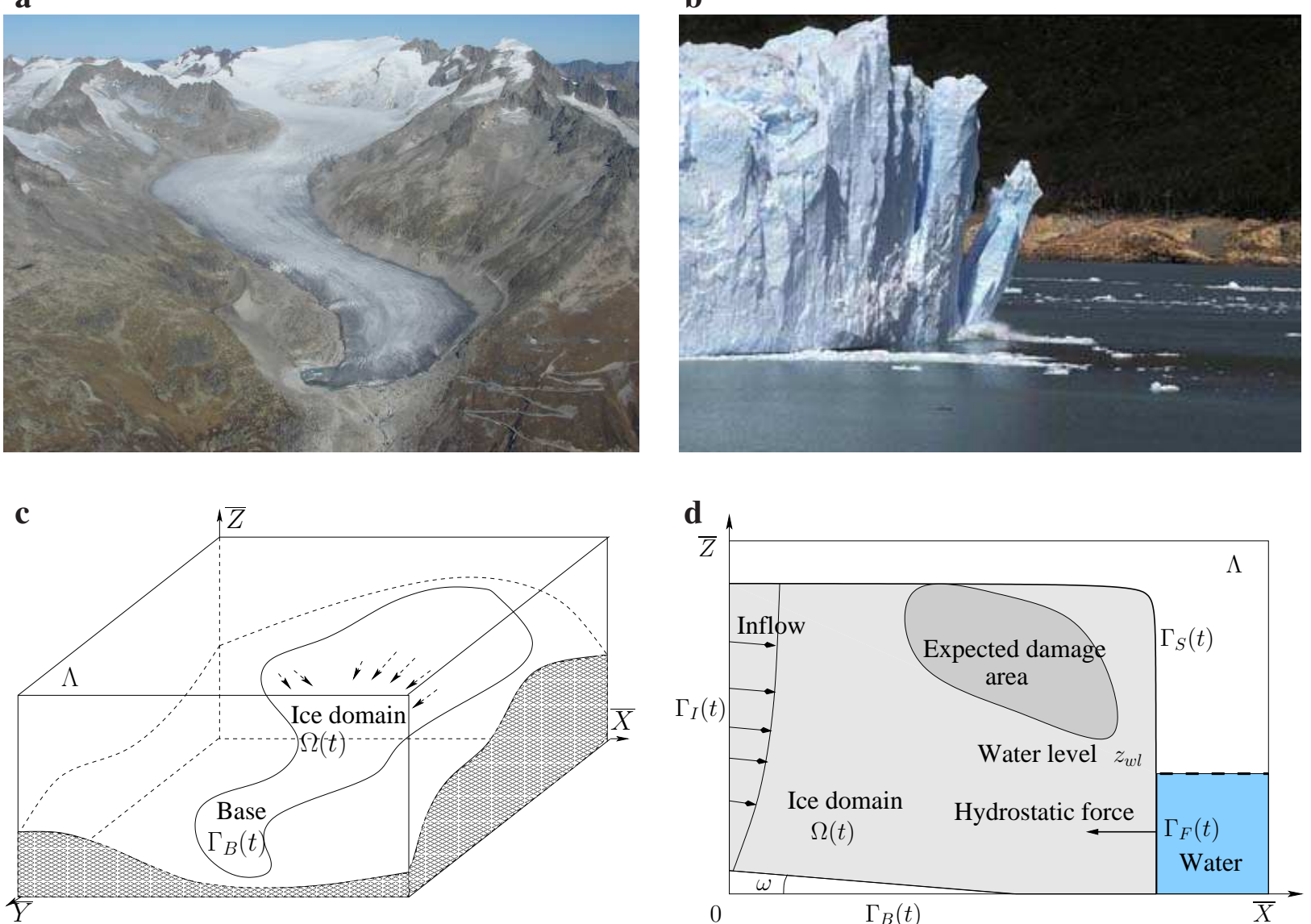

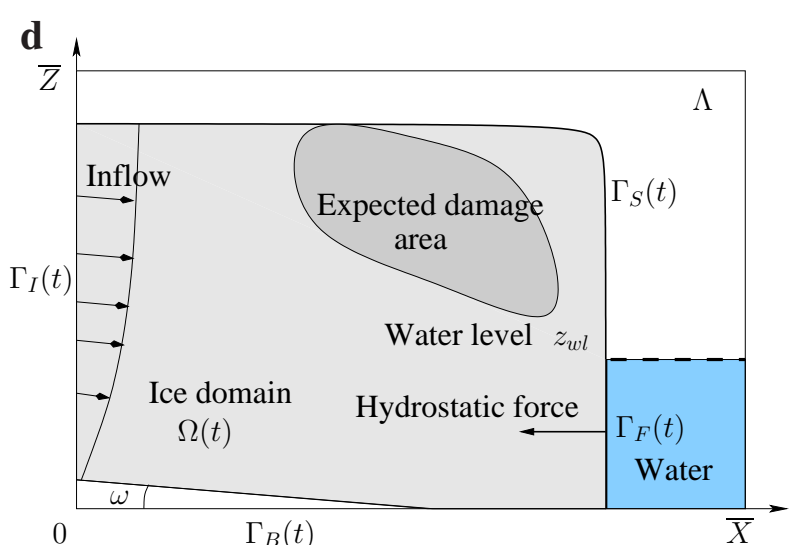

Figure 1: (a) Rhonegletscher in 2008 (source: G. Kappenberger). (b) Ice breaking away occurring during the calving process, picture taken at the front of the Perito Moreno glacier (source: www.firststryke.com). (c) A three dimensional glacier with notations. (d) Section of a glacier's tongue submerged by water with notations.

be solved exactly using the Cardan formula. In practice, Newton's method is used to solve (2.3), which requires less than five iterations to converge, for any value of $m$. The function $\mu$ is displayed with respect to $|\varepsilon(\mathbf{u})|$ in Fig. 2 for fixed parameters $A, m$ and $\tau_{0}$.

\subsection{Damaged glacier velocity}

The evolution of micro-cracks density before failure can be described using continuum damage mechanics [17]. Consider a point $\mathbf{x}$ in the ice region, surrounded by a representative volume element of cross-section $S$. Let $S^{\prime}$ be the cross-sectional area due to micro-cracks, see Fig. 3, damage is a scalar function ranging from zero to one and defined by:

$$
D(\mathbf{x})=\frac{S^{\prime}}{S} .
$$




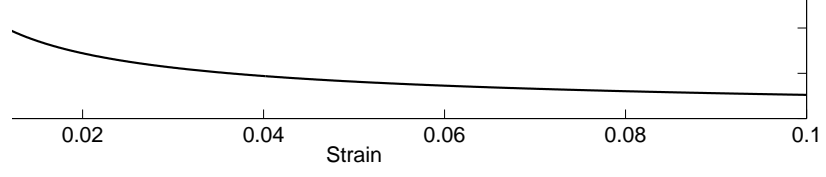

Figure 2: Viscosity (unit: bar year) with respect to the strain's norm $|\varepsilon(\mathbf{u})|$ (unit: year ${ }^{-1}$ ). The parameters are $A=0.08$ bar $^{-3}$ year $^{-1}, m=3$ and $\tau_{0}=\sqrt{0.1}$ bar.

This definition is valid in two or three space dimensions, under the assumption that damage is an isotropic process, that is when the orientation of cracks is uniform in all directions.
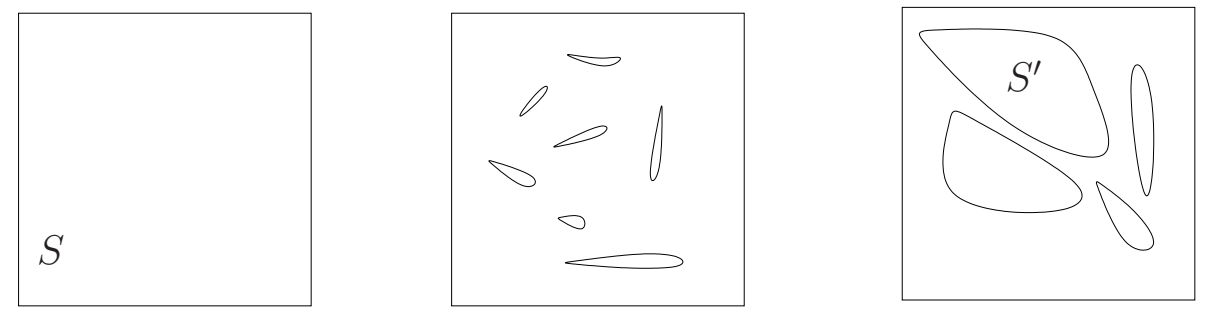

Figure 3: The progressive damage of a material. Left: no damage, $D=0$. Middle and right: partial damage, $D=0.1$ and $D=0.5$. The cross-section of the representative volume element is $S$, while $S^{\prime}$ is the area of the micro-cracks.

When damage increases, the apparent fluid viscosity should decrease, consequently, the Stokes problem with damage writes, see [21]:

$$
\begin{aligned}
-2 \operatorname{div}((1-D) \mu \varepsilon(\mathbf{u}))+\nabla p & =\rho \mathbf{g}, & & \text { in } \Omega(t), \\
\operatorname{div} \mathbf{u} & =0, & & \text { in } \Omega(t),
\end{aligned}
$$

where $\mu$ is still defined by (2.3). This nonlinear Stokes problem supplemented with boundary conditions is well posed whenever the damage $D<1$, which will be the case for the reasons detailed hereafter.

Damage is assumed to be transported with the ice particles, and evolves according to a source term depending on internal stresses, see [21]:

$$
\frac{\partial D}{\partial t}+\mathbf{u} \cdot \nabla D=f(D, \mathbf{u}, p)
$$


Here the source term $f$ is given by:

$$
f(D, \mathbf{u}, p)=B \frac{\max (0, \psi(D, \mathbf{u}, p))^{r}-\lambda \max (0,-\psi(D, \mathbf{u}, p))^{r}}{(1-D)^{k}} \chi_{[0,1-\epsilon]}(D),
$$

where the function $\psi$ is defined by:

$$
\psi(D, \mathbf{u}, p)=\frac{c_{1} 2 \mu|\varepsilon(\mathbf{u})|-c_{2} p}{1-D}-\bar{\sigma} .
$$

Here above $k, \lambda, B, r, \bar{\sigma}, c_{1}$ and $c_{2}$ are positive parameters, $\chi_{[0,1-\epsilon]}(D)$ is the characteristic function of $[0,1-\epsilon]$, one in $[0,1-\epsilon]$ and zero otherwise, $\epsilon$ being a small positive parameter.

The term $\chi_{[0,1-\epsilon]}(D)$ in $(2.8)$ forces the variable $D$ to remain in the interval $[0,1-\epsilon]$, thus avoiding $\mu(1-D)$ to be zero in (2.5). The first term in the top of the fraction in (2.8) is a contribution to damage whereas the second term is a contribution to healing. If $c_{1} 2 \mu|\varepsilon(\mathbf{u})|-c_{2} p>(1-D) \bar{\sigma}$, then damage occurs, otherwise healing occurs.

Remark 1. In (2.9), the term

$$
c_{1} 2 \mu|\varepsilon(\mathbf{u})|-c_{2} p
$$

corresponds to

$$
\alpha \sigma_{1}+\beta \sqrt{3 \tau_{I I}}+(1-\alpha-\beta) \sigma_{I},
$$

appearing in the model of [21], where $\sigma_{1}$ is the maximal eigenvalue of the stress tensor $\sigma, \sigma_{I}:=$ $\operatorname{tr}(\sigma)$ is the first invariant of $\sigma, \tau:=\sigma-\frac{1}{d} \sigma_{I} I$ is the deviatoric stress tensor and $\tau_{I I}:=\frac{1}{2} \tau: \tau$ is the second invariant of $\tau$. Here above, $\alpha$ and $\beta$ are positive parameters such that $1-\alpha-\beta$ is positive. By using the relations $\sigma=2 \mu \varepsilon(\mathbf{u})-p I, \tau=2 \mu \varepsilon(\mathbf{u})$ and $\operatorname{div}(\mathbf{u})=0$, the quantities $\sigma_{1}$, $\tau_{I I}$ and $\sigma_{I}$ can be rewritten as functions of $\varepsilon(\mathbf{u})$ and $p$ :

$$
\sigma_{1}=2 \mu|\varepsilon(\mathbf{u})|-p, \quad \tau_{I I}=(2 \mu|\varepsilon(\mathbf{u})|)^{2}, \quad \sigma_{I}=-d p .
$$

As a consequence, (2.11) is equal to (2.10) with

$$
c_{1}=\alpha+\sqrt{3} \beta, \quad \text { and } \quad c_{2}=\alpha+d(1-\alpha-\beta) .
$$

\subsection{Volume fraction of ice}

In $[20,22]$, the level set approach has been used to describe the changes of the ice domain during the calving process. In this work, another Eulerian description, the Volume of Fluid (VOF) method [23], is preferred.

The volume fraction of ice $\varphi$ is defined inside the cavity $\Lambda$ by:

$$
\varphi(\mathbf{x}, t)=\left\{\begin{array}{cc}
1 & \text { if } \mathbf{x} \in \Omega(t) \\
0 & \text { else }
\end{array}\right.
$$


Let $b(\mathbf{x}, t)$ be the mass balance function, that is the ice thickness added or removed during one year by ice accumulation (snowfalls) and ice ablation (melting). From [15, 16], mass balance yields:

$$
\frac{\partial \varphi}{\partial t}(\mathbf{x}, t)+\mathbf{u} \cdot \nabla \varphi(\mathbf{x}, t)=b(\mathbf{x}, t) \delta_{\Gamma_{S}(t)},
$$

where $\delta_{\Gamma_{S}(t)}$ is the delta Dirac function on the ice-air interface $\Gamma_{S}(t)$ and is defined by:

$$
\int_{V} b \delta_{\Gamma_{S}(t)} d V=\int_{V \cap \Gamma_{S}(t)} b d S
$$

for any domain $V$ in the cavity $\Lambda$.

\subsection{Boundary conditions}

The boundary of $\Omega(t)$ has four components, see Fig. 1d. Basal sliding under the glacier is neglected. Thus, no slip conditions apply along the bedrock-ice interface $\Gamma_{B}(t)$ :

$$
\mathbf{u}=\mathbf{0} \quad \text { on } \Gamma_{B}(t),
$$

while zero force conditions apply on the ice-air interface $\Gamma_{S}(t)$ :

$$
2 \mu \varepsilon(\mathbf{u}) \mathbf{n}-p \mathbf{n}=\mathbf{0} \quad \text { on } \quad \Gamma_{S}(t),
$$

where $\mathbf{n}$ is the unit outer normal. If ice is submerged by water, an hydrostatic pressure applies on the ice-water interface $\Gamma_{F}(t)$ :

$$
2 \mu \varepsilon(\mathbf{u}) \mathbf{n}-p \mathbf{n}=-\rho_{w}|\mathbf{g}|\left(z_{w l}-z\right) \mathbf{n} \quad \text { on } \quad \Gamma_{F}(t),
$$

where $\rho_{w}$ and $z_{w l}$ denote the water density and the water level, respectively. On $\Gamma_{I}(t)$, the part of the boundary where ice enters the cavity $\Lambda$, we have:

$$
\mathbf{u}=\mathbf{u}_{I}, \quad \varphi=1, \quad D=0 \quad \text { on } \quad \Gamma_{I}(t),
$$

where $\mathbf{u}_{I}$ is the given incoming ice flow velocity that satisfies $\mathbf{u}_{I} \cdot \mathbf{n}<0$.

The whole problem consists in finding $\mathbf{u}, p, \varphi$ and $D$ satisfying the partial differential equations (2.5) (2.6) (2.7) (2.15), the boundary conditions (2.16) (2.17) (2.18) (2.19) plus given initial conditions for $\varphi$ and $D$.

The well-posedness of the nonlinear Stokes problem (2.1) (2.2) with boundary conditions (2.16) (2.17) is proved in [14]. The proof relies on the fact that the corresponding weak form is nothing but Euler's equation of a minimization problem, the functional being strictly convex due to $(2.4)$.

\section{Numerical method}

A time discretization scheme is proposed to decouple the transport problems (2.7) (2.15) and the nonlinear Stokes problem (2.5) (2.6), as in [18, 5, 3, 16]. This allows transport and Stokes problems to be solved on two different grids by using different numerical techniques. 


\subsection{Time discretization}

Let $0=t^{0}<t^{1}<t^{2}<\ldots<t^{N}=T$ be a subdivision of the time interval $[0, T]$, and $\tau^{n}=t^{n}-t^{n-1}$ be the $n$-th time step, $n=1,2, \ldots, N$. The largest time step is denoted by $\tau_{\max }$. Assume that $\left(\varphi^{n-1}, \mathbf{u}^{n-1}, p^{n-1}, D^{n-1}\right)$, approximations of $(\varphi, \mathbf{u}, p, D)$ at time $t^{n-1}$, are known. We now detail how to compute $\left(\varphi^{n}, \mathbf{u}^{n}, p^{n}, D^{n}\right)$.

The two advection problems (2.7) and (2.15) are solved using a one order splitting scheme to decouple the advection and source terms. The first step consists in solving the two following transport problems between $t^{n-1}$ and $t^{n}$ :

$$
\left\{\begin{array} { l l } 
{ \frac { \partial \varphi } { \partial t } + \mathbf { u } ^ { n - 1 } \cdot \nabla \varphi } & { = 0 , } \\
{ \varphi ( t ^ { n - 1 } ) } & { = \varphi ^ { n - 1 } , }
\end{array} \quad \left\{\begin{array}{ll}
\frac{\partial D}{\partial t}+\mathbf{u}^{n-1} \cdot \nabla D & =0, \\
D\left(t^{n-1}\right) & =D^{n-1},
\end{array}\right.\right.
$$

with boundary conditions (2.19). A one order approximation of the solution of (3.1) at time $t^{n}$, denoted by $\left(\varphi^{n-\frac{1}{2}}, D^{n-\frac{1}{2}}\right)$, can be obtained using the forward method of characteristics:

$$
\begin{aligned}
\varphi^{n-\frac{1}{2}}\left(\mathbf{x}+\tau^{n} \mathbf{u}^{n-1}(\mathbf{x})\right) & =\varphi^{n-1}(\mathbf{x}), \\
D^{n-\frac{1}{2}}\left(\mathbf{x}+\tau^{n} \mathbf{u}^{n-1}(\mathbf{x})\right) & =D^{n-1}(\mathbf{x}) .
\end{aligned}
$$

The ice region is then defined by:

$$
\Omega^{n-\frac{1}{2}}=\left\{\mathbf{x} \in \Lambda ; \varphi^{n-\frac{1}{2}}(\mathbf{x})=1\right\}
$$

and the ice-air interface $\Gamma_{S}^{n-\frac{1}{2}}$ can be identified. The second step consists in solving the two next problems between $t^{n-1}$ and $t^{n}$ :

$$
\left\{\begin{array} { l l } 
{ \frac { \partial \varphi } { \partial t } } & { = b ( \cdot , t ^ { n - 1 } ) \delta _ { \Gamma _ { S } ^ { n - \frac { 1 } { 2 } } } , } \\
{ \varphi ( t ^ { n - 1 } ) } & { = \varphi ^ { n - \frac { 1 } { 2 } } }
\end{array} \quad \left\{\begin{array}{ll}
\frac{\partial D}{\partial t} & =f\left(D^{n-1 / 2}, \mathbf{u}^{n-1}, p^{n-1}\right) \\
D\left(t^{n-1}\right) & =D^{n-\frac{1}{2}}
\end{array}\right.\right.
$$

Finally, an order one approximation of $\varphi$ and $D$ at time $t^{n}$ is given by:

$$
\begin{aligned}
\varphi^{n} & =\varphi^{n-1 / 2}+\tau^{n} b\left(\mathbf{x}, t^{n-1}\right) \delta_{\Gamma_{S}^{n-\frac{1}{2}}} \\
D^{n} & =\max \left(\min \left(D^{n-1 / 2}+\tau^{n} f\left(D^{n-1 / 2}, \mathbf{u}^{n-1}, p^{n-1}\right), 1-\epsilon\right), 0\right),
\end{aligned}
$$

so that the computed damage $D^{n}$ clearly remains between 0 and $1-\epsilon$. The new ice domain $\Omega^{n}$ is defined as in (3.4) with $\varphi^{n}$ in place $\varphi^{n-\frac{1}{2}}$. The incoming-flow interface $\Gamma_{I}^{n}$, the bedrock-ice interface $\Gamma_{B}^{n}$, the water-ice interface $\Gamma_{F}^{n}$ and the ice-air interface $\Gamma_{S}^{n}$ can then be identified. Finally, the weak formulation corresponding to the nonlinear Stokes problem (2.5) (2.6) consists in finding $\mathbf{u}^{n}: \Omega^{n} \rightarrow \mathbb{R}^{d}$ and $p^{n}: \Omega^{n} \rightarrow \mathbb{R}$ such that $\mathbf{u}^{n}=\mathbf{0}$ on $\Gamma_{B}^{n}, \mathbf{u}^{n}=\mathbf{u}_{I}$ on $\Gamma_{I}^{n}$, and

$$
\begin{aligned}
2 \int_{\Omega^{n}}\left(1-D^{n}\right) \mu\left(\left|\varepsilon\left(\mathbf{u}^{n}\right)\right|\right) \varepsilon\left(\mathbf{u}^{n}\right): & \varepsilon(\mathbf{v}) d V-\int_{\Omega^{n}} p^{n} \operatorname{div} \mathbf{v} d V+\int_{\Omega^{n}} \operatorname{div} \mathbf{u}^{n} q d V \\
& \quad-\rho \int_{\Omega^{n}} \mathbf{g} \cdot \mathbf{v} d V+\rho_{w}|\mathbf{g}| \int_{\Gamma_{F}^{n}}\left(z_{w l}-z\right)(\mathbf{v} \cdot \mathbf{n}) d S=0,
\end{aligned}
$$

for all $(\mathbf{v}, q)$ such that $\mathbf{v}=\mathbf{0}$ on $\Gamma_{B}^{n} \cup \Gamma_{I}^{n}$. 


\subsection{A two-grid method for space discretization}

Two fixed meshes are used for solving the advection and diffusion problems, see [18, 4, 3, 16]. Formula (3.2) (3.3) (3.6) (3.7) are implemented on a fixed structured grid $\mathcal{T}_{h}$ made of small cells having size $h$, with goal to reduce the numerical diffusion of $\varphi$ as much as possible. As the bedrock topography can be rather complex, the Stokes problem (3.8) is solved using a fixed unstructured mesh $\mathcal{T}_{H}$ of tetrahedrons with typical size $H$, see Fig. 4. Note that $\mathcal{T}_{H}$ can be non uniform, see the numerical results of section 5; in that case, $H$ is defined to be the smallest size of the tetrahedrons. Since most of the CPU time is spent for solving Stokes problem, $H$ is larger than $h$. A trade-off between accuracy and efficiency is to choose $H \simeq 5 h$, see [18].

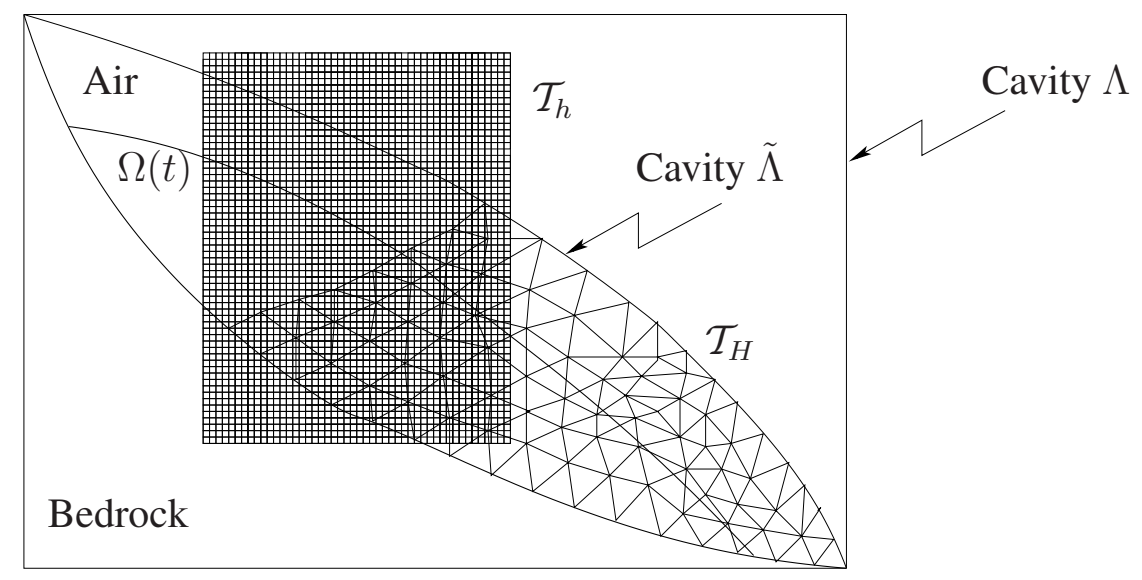

Figure 4: Two dimensional example of the space discretisation: $\mathcal{T}_{H}$ is an unstructured mesh that fits the bedrock topography while $\mathcal{T}_{h}$ is a grid overlapping $\mathcal{T}_{H}$.

The implementation of the formula (3.2) and (3.3) is an easy task on the structured mesh $\mathcal{T}_{h}$. For each cell, $\varphi^{n-1}$ and $D^{n-1}$ are advected according to $\mathbf{u}^{n-1}$, and then projected onto the grid $\mathcal{T}_{h}$, see $[18,16]$. Numerical diffusion in the variable $\varphi$ is introduced during the projection step and the volume fraction of ice is smeared, taking values between zero and one. The use of the SLIC algorithm (Simple Line Interface Calculation) allows the width of the diffusion layer to be reduced at low cost, see for instance [23]. On the other hand, the damage variable $D$ varies smoothly but rapidly from 0 to $1-\epsilon$ and thus does not require the use of the SLIC algorithm.

This transport algorithm is unconditionally stable and CFL numbers greater than one can be used. The use of an adaptive time stepping is advocated in order to capture rapid changes in the damage variable $D$. The current time step is computed using the formula:

$$
\tau^{n}=\min \left(\frac{\overline{\mathrm{CFL}} \times h}{\left\|\mathbf{u}^{n}\right\|_{L^{\infty}\left(\Omega^{n}\right)}}, \tau_{\max }\right),
$$

where $\overline{\mathrm{CFL}}$ and $\tau_{\max }$ are two given parameters, representing the maximal CFL number and the maximal time step, respectively. 
The diffusion step consists in solving the Stokes problem (3.8) with standard finite element techniques on the mesh of tetrahedrons $\mathcal{T}_{H}$. The use of unstructured tetrahedrons allows mesh refinement whenever needed, crevasse formation for instance. Moreover, the tetrahedrons can be distorded in order to have a finer mesh spacing along the vertical directions. Continuous, piecewise linear stabilized finite elements are used as in [7]. A fixed point method is used to solve the nonlinearity due to the viscosity, we refer to $[18,5,3,16]$ for details about the diffusion step. A priori error estimates for the finite element approximation corresponding to (3.8) in a prescribed domain are established in [14], using a quasi-norm technique [1]. Convergence of the fixed point method is also discussed.

\section{Three dimensional simulation of Rhonegletscher}

Rhonegletscher is a medium-sized alpine valley glacier with a well developed tongue. It has a length of about $8 \mathrm{~km}$, and an area of more than $15 \mathrm{~km}^{2}$. The current ice volume is $2 \mathrm{~km}^{3}$ [6]. The ice velocity at the surface reaches up to 100 meters per year [15]. As most Alpine glaciers, Rhonegletscher has significantly retreated since the end of the 19th century, and has decreased about 1.2 $\mathrm{km}$ in length since 1880 [8]. The numerical simulation of this glacier from 1874 to 2007 has reproduced observations with accuracy in [15]. Projections of the glacier retreat until 2100 have been performed according to three realistic climate change scenarios based on the Swiss Advisory Body on Climate Change, see occc. ch [15]. The goal of the present experiment is to explore other simple scenarios that are independent of predictions based on climate model results. Projections of climate over the next century show a large spread and are thus controversial. This is both due to unknown future anthropogenic contribution and to climate model uncertainty. Therefore, we define three scenarios that are based on observed climate conditions in the past, and just repeat these over the entire modelling period 2007-2100.

- Scenario 'cold' is obtained by driving the model with air temperature and precipitation between October 1977 and September 1978, which resulted in the most positive annual mass balance of glaciers in the European Alps during last century [12].

- Scenario 'current' is obtained by randomly picking one year between 2000 and 2008 that provides the climatic forcing. This is repeated for all years until 2100.

- Scenario 'hot' is based on the meteorological conditions between October 2002 and September 2003 leading to extremely negative mass balance of Alpine glaciers. This year could be a precursor of the coming decades.

The bedrock topography of Rhone glacier is reconstructed from measurements [6], and the elevation of the ice surface is available for 2007. From these data, the meshes $\mathcal{T}_{H}$ and $\mathcal{T}_{h}$ can be generated as described in [15]. The size of the final meshes is: $H \sim 50$ meters and $h=10$ meters. The time step is constant $\tau^{n}=0.5$ year. Glen's exponent is set to $m=3$, see [11] and the regularisation parameters are fixed $\tau_{0}=\sqrt{0.1}$ bar. As in [15], sliding effects are neglected 
over the period 2007-2100. The rate factor $A$ has been calibrated such that it minimizes the meansquare error between computed and measured ice surface velocities, see [15]. As a result, we set to $A=0.08 \operatorname{bar}^{-3} \mathrm{y}^{-1}$ over the period 2007-2100. Since no ice enters the cavity $\Lambda$, we set $\Gamma_{I}(t)=\emptyset$. Moreover, we assume that the glacier does not reach water, thus $\Gamma_{F}(t)=\emptyset$. At the glacier surface a mass balance function $b$ is prescribed to incorporate climatic forcing. $b$ relies, among other variables, on measured daily air temperature and precipitation and has been determined for Rhone glacier in previous studies [12]. The model is driven using the three scenarios based on observed meteorological conditions in the past (see above). The numerical simulations start in 2007 and end in 2010. Since the whole glacier is considered, damage can be neglected, thus $D=0$.

Snapshots and total ice volumes of the three simulations corresponding to the different scenarios are displayed in Fig. 4. According to Scenario 'current', Rhonegletscher will continue to decay and lose $36 \%$ of the total ice volume by 2100 compared to 2007 . This indicates that the size of the glacier is too large to be stable in the current climate conditions. Thus, Rhonegletscher will further retreat, also if future climate stabilizes on the level of the last decade. The simulation based on Scenario 'hot' results in an almost complete disappearance of the glacier by 2100 . Only $11 \%$ of the total ice volume will remain in this case. This underlines the extreme impact of the summer heat waves of the year 2003 on the Alpine glaciers. If conditions like in 2003 prevail in the future decades, glaciers in the European Alps would have bad chances of surviving. According to Scenario 'cold', however, Rhonegletscher could expand its size rapidly. In 2085, the ice volume would be almost be multiplied by two (compared to 2007), and the glacier would be larger than during the maximum of the Little Ice Age 150 years ago. Note that the simulation has finished in 2085 since the glacier has extended beyond the cavity. This experiment shows that meteorological conditions in individual years can be very favourable to glaciers. Indeed, many glaciers showed significant advances in the 1970s [8], which were however stopped by rapidly rising air temperatures after the mid-1980s.

\section{Two dimensional simulation of calving}

Break-off of ice and calving processes are often observed phenomenon on many glaciers worldwide. Calving is especially important for the prediction of the dynamics of glaciers terminating in the ocean (e.g. Antarctica or Greenland) [2], whereas break-off of ice blocks from hanging glaciers in the Alps can be a serious natural hazard [21]. Calving contributes of mass loss from the continental ice sheets and therefore has the potential to rise the sea level [2]. The modelling of these processes is highly important in order to anticipate some natural events such that the sudden glacier rupture or a the sea level rise, and has a large potential for application in numerous glaciological studies.

Pralong and Funk have demonstrated in [21] the ability of the local damage evolution law (2.7) applied to Glen's law to model the breaking-off of ice masses from glaciers. Good agreements between observations and simulations have confirmed the relevance of the model. However, their approach is based on a level set description of the glacier geometry and requires a remeshing procedure, see [19], which may artificially influence the behaviour of cracks. Moreover, the mesh- 
dependence of their results has not been investigated.

Here, we present a simulation of the calving of a two-dimensional glacier. The study case is inspired from the simulation of Gruben glacier presented in [21] but can also represent the end of any glacier terminating in water. We consider the terminus of a glacier flowing from left to right and finishing with a vertical ice front against a lake. The bedrock contains two flat parts: a slightly inclined part at an angle $\omega$ on the glacier (left) side and an horizontal part on the water (right) side, see Fig. 1d. The presence of water at glacier margins induces a force on the ice-water interface due to hydrostatic pressure. Since calving results from local damage effects of ice, there is no need to consider the entire volume of ice. The glacier is then truncated, see Fig. 1d, and the incoming ice flow at $\Gamma_{I}(t)$ is given by the formula:

$$
\mathbf{u}_{I}(z)=\left(\begin{array}{c}
\cos (\omega) \\
-\sin (\omega)
\end{array}\right) \bar{C}\left(S^{m+1}(0)-(S(0)-z)^{m+1}\right)
$$

where $S(x)$ is the ice thickness, $\omega$ is the angle of inclination of the inflow-side bedrock, and $\bar{C}>0$. Note that the velocity profile given by (5.1) is an exact solution of the Shallow Ice Approximation equation, see $[13,10]$.

At initial time, a nearly rectangular ice domain is considered, see Fig. 1d, damage being zero. The physical and numerical parameters used in the paper are reported in Table 1. The parameters $B, r, k$ and $\lambda$ arising in equations (2.8) (2.9) are taken from [19], see [21] for more details concerning the calibration procedure. The parameters $c_{1}$ and $c_{2}$ are deduced from the values of $\alpha$ and $\beta$ given in [19] via the relations (2.13). The coefficient $\bar{\sigma}$ is computed according to equation (18) of [21]. The parameters $\varepsilon$ and $\tau_{\max }$ are taken from [14]. The coefficient $\bar{C}$ is chosen so that the maximum of the velocity profile is about 2.5 meters per year. Since the simulation time is only one year, ice accumulation and ablation is neglected, i.e. $b=0$. The mesh $\mathcal{T}_{h}$ is a grid with $600 \times 800$ squared cells overlapping the cavity $\Lambda$ while the mesh $\mathcal{T}_{H}$ contains about 16,000 nodes which are not uniformly distributed. Indeed, $\mathcal{T}_{H}$ is finer in the expected damaged zone, see Fig. $1 \mathrm{~d}$, and coarser elsewhere in order to save memory and to reduce the computational time. The size of the smallest triangle is about $0.25 \mathrm{~m}$. With meshes $\mathcal{T}_{h}$ and $\mathcal{T}_{H}$, the recommended ratio $\frac{H}{h} \sim 5$ holds in the finest zone and is higher elsewhere. Unlike Section 4, the time unit is a second.

The choice of $\epsilon$ (here, taken to be $10^{-3}$ ) is delicate. Indeed, if $\epsilon$ is too small, then the matrix corresponding to (2.5) (2.6) becomes ill-conditioned. Since the problem is in two space dimensions, a direct solver can be used and fails only when $\epsilon<10^{-5}$.

The evolution of ice calving is presented in Fig. 6. Until day 172, the ice flow is very slow and the time step - computed using (3.9) - equals $\tau_{\max }$. Then, the stress and the pressure are such that damage initiates and propagates rapidly. A crevasse appears in the most damaged region and an ice block breaks apart. The crack propagates, the damaged zone behaves as a thin viscous liquid layer and the motion of ice increases. Due to (3.9), the time step significantly decreases. When the ice block detaches from the glacier, it falls down and exits the cavity $\Lambda$.

The influence of the mesh size on the result is investigated. Three meshes $\mathcal{T}_{H}$ are considered with several levels of refinement: meshes 1, 2 and 3, respectively, from the coarsest one to the finest one. The results displayed in Fig. 6 have been obtained by using mesh 3. For any mesh, the main crack initializes at the same location and propagates in the same direction with a comparable size, 
see Fig. 7. Nevertheless, the finest mesh displays small cracks that are ignored by the two coarsest meshes. For meshes 1, 2 and 3, the main crack initializes at 244, 183 and 172 days, respectively, thus the time of failure seems to converge with mesh size.

This study shows that the volume fraction of ice description is a relevant alternative to the level set description used in [21]. Unlike the method proposed by Pralong and Funk, no remeshing is needed. Moreover, the behaviour and the location of the cracks inside the ice domain are not very sensitive to the mesh size.

\begin{tabular}{|c|c|c|}
\hline Parameter & Value & Unit \\
\hline$m$ & 3 & - \\
\hline$A$ & $6.8 \times 10^{-24}$ & $\mathrm{~Pa}^{-m} \mathrm{~s}^{-1}$ \\
\hline$|\mathbf{g}|$ & 9.81 & $\mathrm{~m} \mathrm{~s}^{-2}$ \\
\hline$\rho$ & 900 & $\mathrm{~kg} \mathrm{~m}^{-3}$ \\
\hline$\rho_{w}$ & 1000 & $\mathrm{~kg} \mathrm{~m}^{-3}$ \\
\hline$B$ & $1.7 \times 10^{-9}$ & $\mathrm{~Pa}^{-r} \mathrm{~s}^{-1}$ \\
\hline$c_{1}$ & 1.3 & - \\
\hline$c_{2}$ & 0.53 & - \\
\hline $\bar{X}$ & 45 & $\mathrm{~m}$ \\
\hline $\bar{Z}$ & 35 & $\mathrm{~m}$ \\
\hline $\bar{C}$ & $10^{-13}$ & $\mathrm{~ms}$ \\
\hline
\end{tabular}

\begin{tabular}{|c|c|c|}
\hline Parameter & Value & Unit \\
\hline$\lambda$ & 0.4 & - \\
\hline$r$ & 0.43 & - \\
\hline$k$ & 2 & - \\
\hline $\bar{\sigma}$ & 44000 & $\mathrm{~Pa}$ \\
\hline$z_{w l}$ & 12 & $\mathrm{~m}$ \\
\hline$\tau_{0}$ & 1000 & $\mathrm{~Pa}$ \\
\hline$\tau_{\max }$ & $10^{5}$ & $s$ \\
\hline$\epsilon$ & $10^{-3}$ & - \\
\hline$\overline{\mathrm{CFL}}$ & 0.9 & - \\
\hline$\omega$ & 4.2 & $\mathrm{Deg}$ \\
\hline- & - & - \\
\hline
\end{tabular}

Table 1: Physical and numerical parameters.

\section{Conclusions}

A physical model has been presented to describe the dynamics of glaciers with the local damage effects that may drive ice to break. Given the ice domain, a nonlinear Stokes problem has to be solved to obtain the velocity of ice. Whenever needed, a damage variable describes continuously the presence of cracks. The damage function satisfies a transport equation with source terms. Then, the viscosity of ice must be updated accordingly. A Eulerian formulation based on the volume fraction of ice is used to describe the evolution of the ice domain. The volume fraction of ice satisfies a transport equation with source terms on the ice-air interface to account for accumulation or melting. Two different grids are used to solve the Stokes and transport equations.

This model allows complex geometries with possible changes of topology to be handled. Two applications demonstrate the potential of the numerical model for simulating glaciological processes. First, the simulation of Rhonegletscher is performed from 2007 to 2100 using three climatic scenarios, but neglecting damage. The results predict that Rhonegletscher will continue to retreat even if the climate of the last decade persists over the entire $21^{\text {th }}$ century and no additional warming occurs. Second, a two dimensional simulation of the calving process is presented. Damage is considered and the opening of a crevasse is observed. Since the method does not require any 
remeshing procedure, mesh effects remain limited. Based on the results shown we recommend the proposed method for numerical simulation of future glacier retreat and the prediction of break-off of ice masses.

\section{Acknowledgements}

The first author is supported by the Swiss National Science Foundation. The authors thank Antoine Pralong for valuable discussions concerning the damage model. Andreas Bauder and Daniel Farinotti are acknowledged for providing the bedrock topography and glacier elevation of Rhonegletscher. This work was funded by the Swiss National Science Foundation, Project No. 200021119721.

\section{References}

[1] J. W. Barrett, W. B. Liu. Quasi-norm error bounds for the finite element approximation of a non-Newtonian flow. Numer. Math. 68 (1994), no. 4, 437-456.

[2] D. I. Benn, C. R. Warren, R. H. Mottram. Calving processes and the dynamics of calving glaciers. Earth-Science Reviews, 82 (2007), no. 3-4, 143 - 179.

[3] A. Bonito, M. Picasso, M. Laso. Numerical simulation of 3D viscoelastic flows with free surfaces. J. Comput. Phys., 215 (2006), no. 2, 691-716.

[4] A. Caboussat, G. Jouvet, M. Picasso, J. Rappaz. Numerical algorithms for free surface flow. Book chapter in CRC volume 'Computational Fluid Dynamics' (2011).

[5] A. Caboussat, M. Picasso, J. Rappaz. Numerical simulation of free surface incompressible liquid flows surrounded by compressible gas. J. Comput. Phys., 203 (2005), no. 2, 626-649.

[6] D. Farinotti, M. Huss, A. Bauder, M. Funk, M. Truffer, A method to estimate ice volume and ice thickness distribution of alpine glaciers. J. Glaciol., 55 (2009), no. 191, 422-430.

[7] L. P. Franca, S. L. Frey. Stabilized finite element methods. II. The incompressible NavierStokes equations. Comput. Methods Appl. Mech. Engrg., 99 (1992), no. 2-3, 209-233.

[8] The Swiss Glaciers, 1880-2006/07. Tech. Report 1-126, Yearbooks of the Cryospheric Commission of the Swiss Academy of Sciences (SCNAT), 1881-2009, Published since 1964 by Laboratory of Hydraulics, Hydrology and Glaciology (VAW) of ETH Zürich.

[9] J.W. Glen. The flow law of ice.. IUGG/IAHS Symposium of Chamonix IAHS Publication, 47 (1958), 171183.

[10] R. Greve, H. Blatter. Dynamics of ice sheets and glaciers. Springer Verlag, 2009. 
[11] G.H. Gudmundsson. A three-dimensional numerical model of the confluence area of unteraargletscher, Bernese Alps, Switzerland. J. Glaciol., 45 (1999), no. 150, 219-230.

[12] M. Huss, A. Bauder, M. Funk, R. Hock. Determination of the seasonal mass balance of four alpine glaciers since 1865. Journal of Geophysical Research, 113 (2008).

[13] K. Hutter. Theoretical glaciology. Reidel, 1983.

[14] G. Jouvet. Modélisation, analyse mathématique et simulation numérique de la dynamique des glaciers. Ph.D. thesis, EPF Lausanne, 2010.

[15] G. Jouvet, M. Huss, H. Blatter, M. Picasso, J. Rappaz. Numerical simulation of rhonegletscher from 1874 to 2100 . J. Comp. Phys., 228 (2009), 6426-6439.

[16] G. Jouvet, M. Picasso, J. Rappaz, H. Blatter. A new algorithm to simulate the dynamics of a glacier: theory and applications. J. Glaciol., 54 (2008), no. 188, 801-811.

[17] J. Lemaitre. A course on damage mechanics. Springer, 1992.

[18] V. Maronnier, M. Picasso, J. Rappaz. Numerical simulation of three-dimensional free surface flows. Internat. J. Numer. Methods Fluids, 42 (2003), no. 7, 697-716.

[19] A. Pralong. On the instability of hanging glaciers. Ph.D. thesis, ETH Zurich, 2005.

[20] A. Pralong, M. Funk, A level-set method for modeling the evolution of glacier geometry. J. Glaciol., 50 (2004), no. 171, 485-491.

[21] A. Pralong, M. Funk. Dynamic damage model of crevasse opening and application to glacier calving. J. Geophys. Res., 110 (2005).

[22] A. Pralong, M. Funk, M. Lüthi. A description of crevasse formation using continuum damage mechanics. Ann. Glaciol., 37 (2003), no. 1, 77-82.

[23] R. Scardovelli, S. Zaleski. Direct numerical simulation of free-surface and interfacial flow. Ann. Rev. Fluid Mech., 31 (1999), no. 7, 567-603.

[24] A. Zryd. Les glaciers en mouvement. Presses polytechniques et universitaires romandes, 2008. 
2007 (initialization)

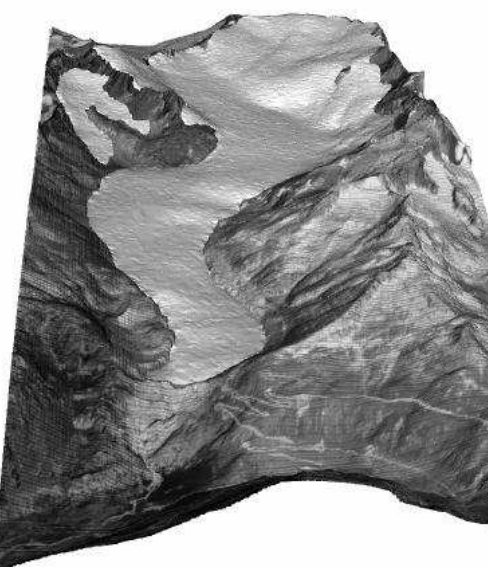

Scenario 'cold'

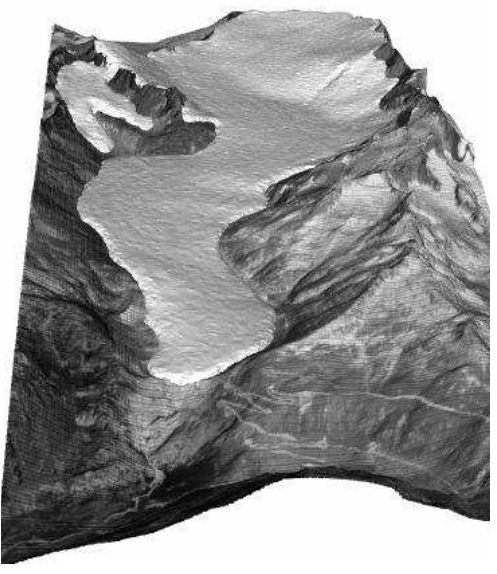

2050

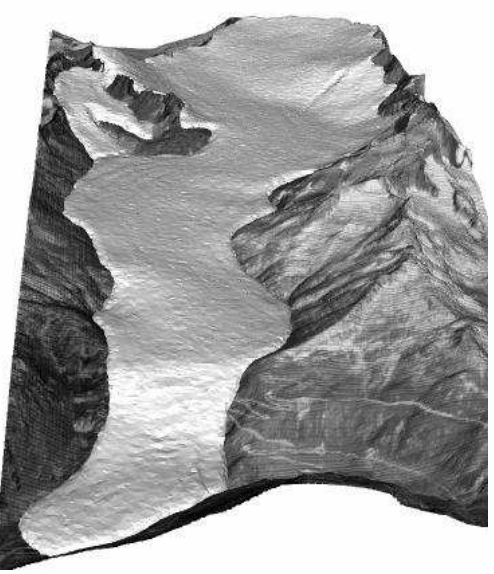

2085
Scenario 'current'

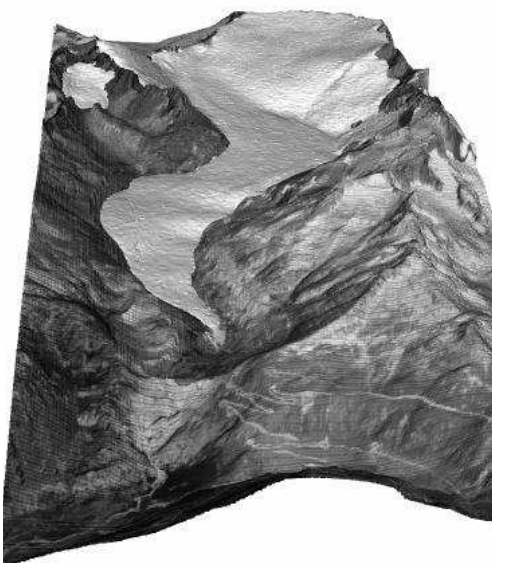

2050

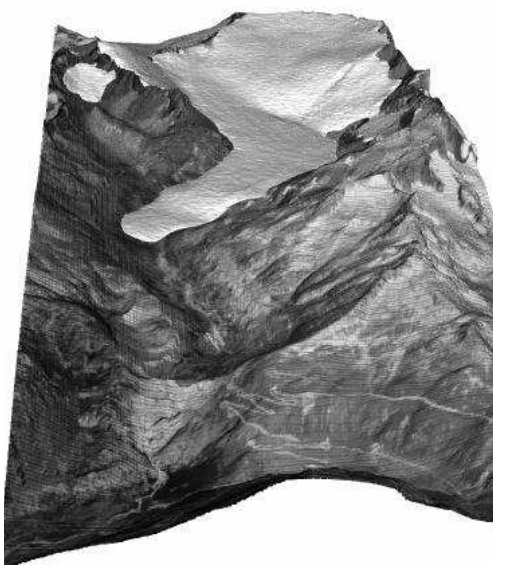

2100

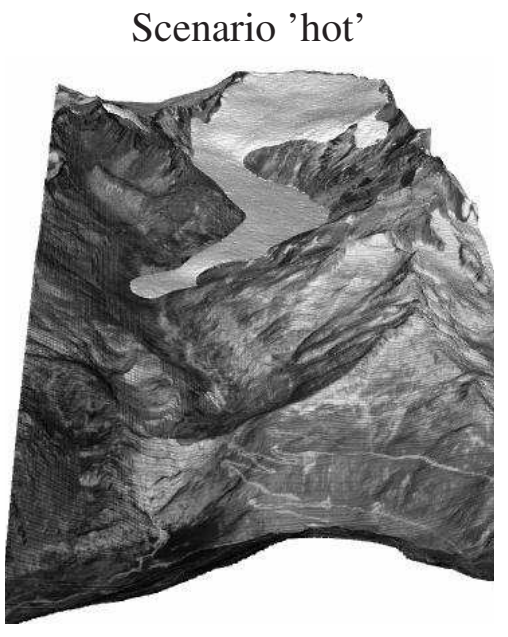

2050

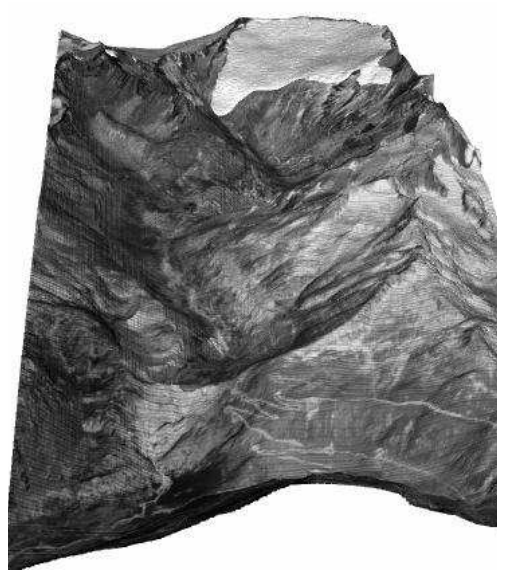

2100

Figure 5: Top left: initialization, Rhonegletscher in 2007. Top right: total ice volume of Rhonegletscher with respect to time according to the different scenarios. Bottom: simulation of Rhonegletscher according to different scenarios. 

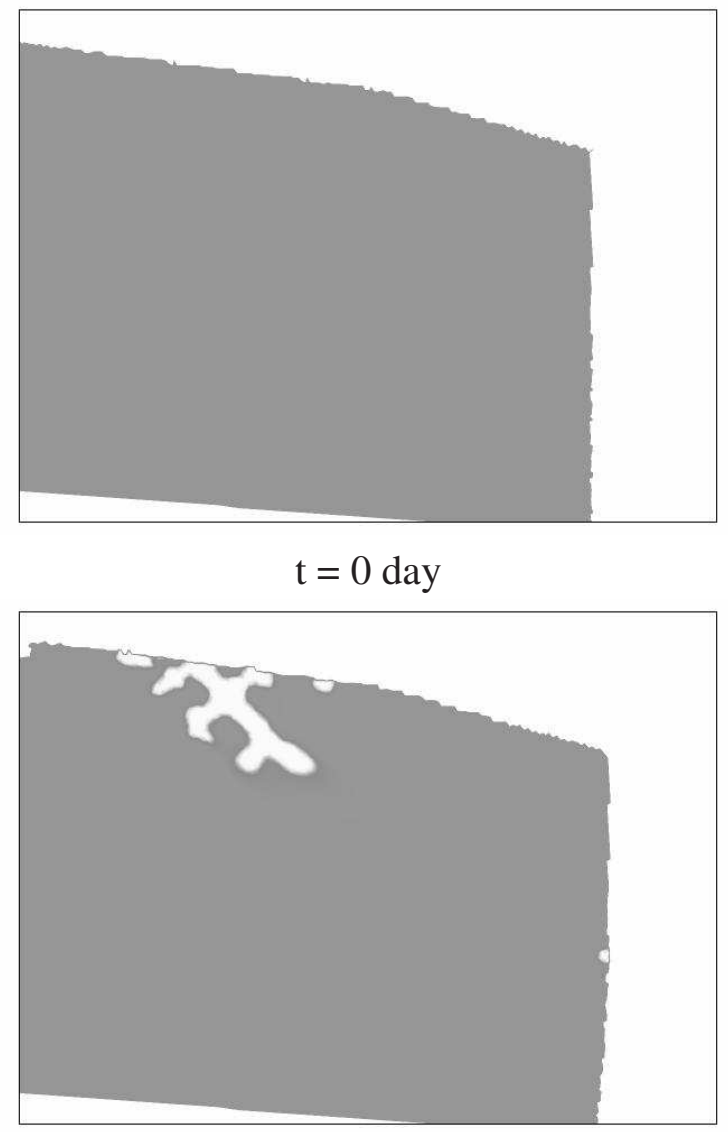

$\mathrm{t}=120$ days

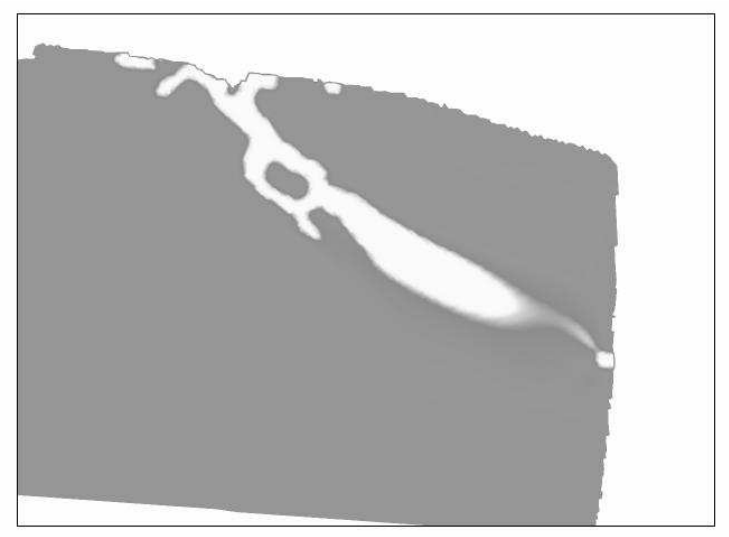

$\mathrm{t}=172.134$ days

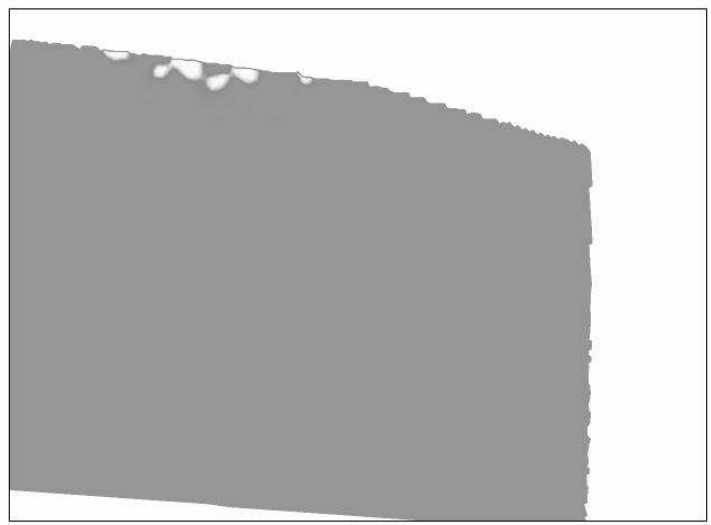

$\mathrm{t}=50$ days

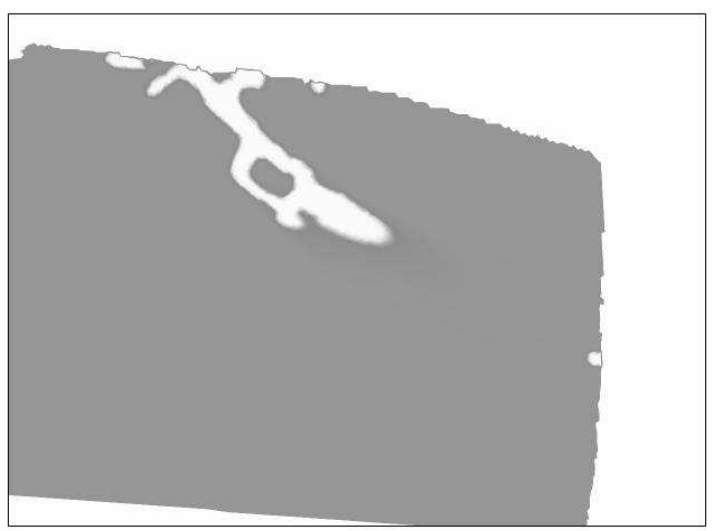

$\mathrm{t}=155$ days

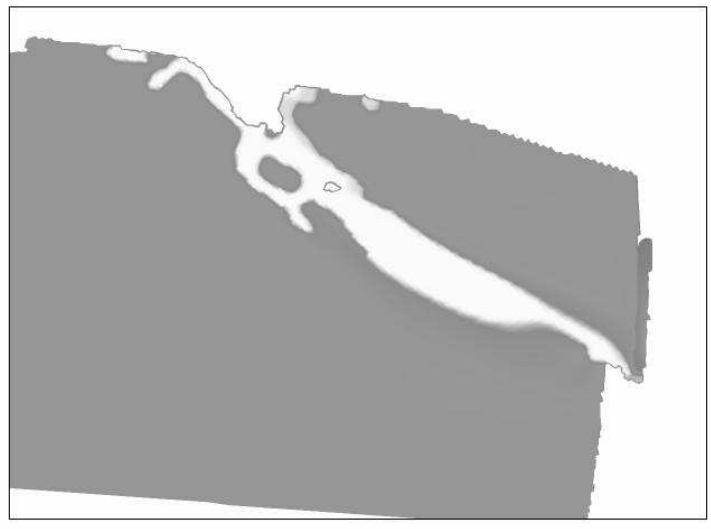

$\mathrm{t}=172.513$ days

Figure 6: Numerical simulation of the calving process. Gray color represents undamaged ice $(D=0)$ while white color represents the maximum of damage $(D=1-\epsilon)$ and air. Time $t$ is displayed in days. 

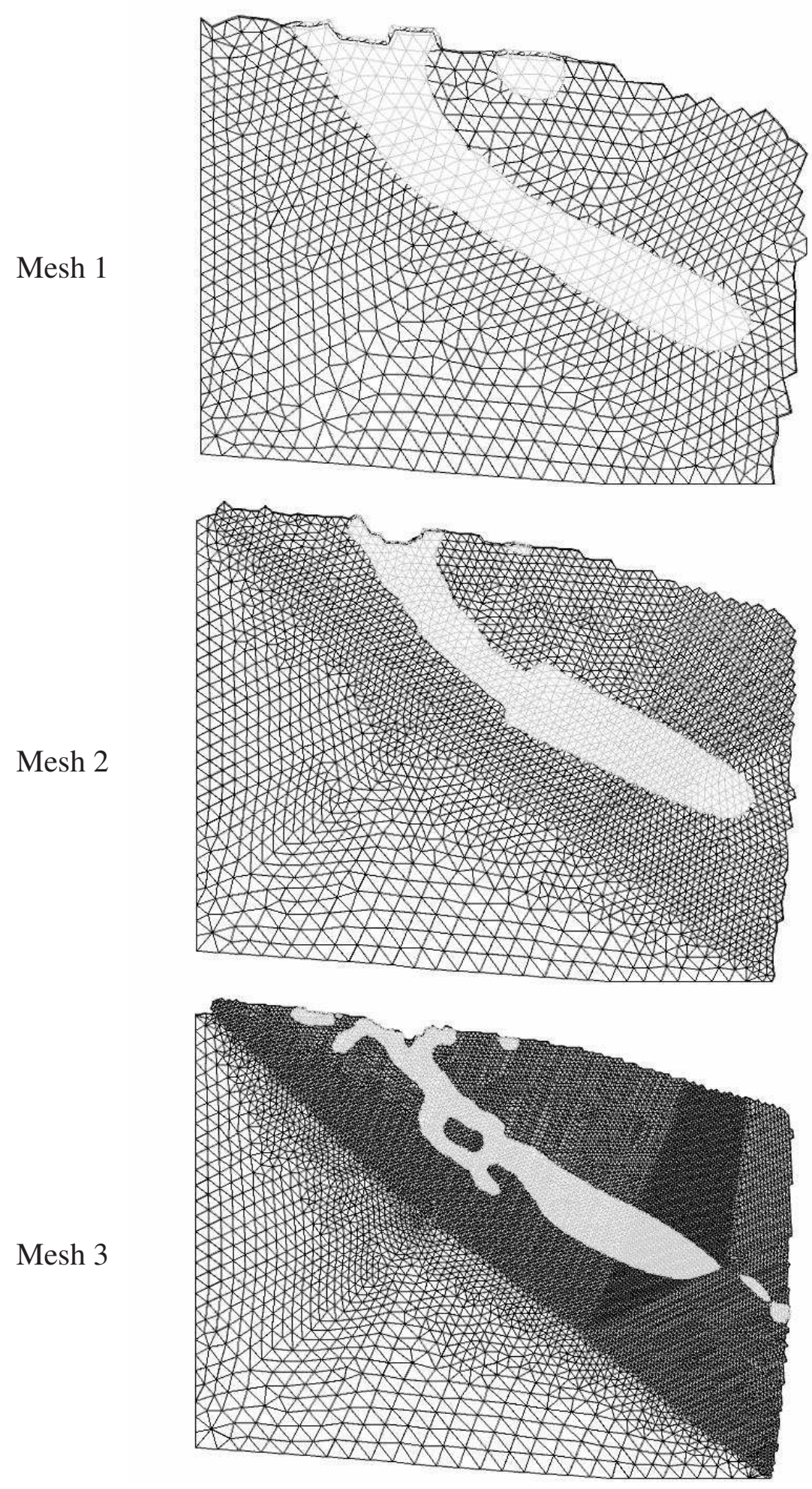

Figure 7: Damage field represented by the white color on meshes 1,2 and 3 about one day before the failure. 